\title{
Autoeficacia y actitud hacia el consumo de drogas en la infancia: explorando los conceptos
}

\author{
Dora Stella Melo Hurtado ${ }^{1}$ \\ Lucila Castanheira Nascimento
}

\begin{abstract}
El objetivo de este artículo es explorar los conceptos de autoeficacia y actitud hacia el consumo, los que fueron analizados bajo la teoría de la motivación y la conducta humana. Se trata de una reflexión teórica, construida con base en la literatura. Altos niveles de autoeficacia tienen consecuencias beneficiosas para el funcionamiento del individuo. Frente a la actitud hacia las drogas se encontró que la presencia o ausencia de prejuicios y las valoraciones positivas sobre las drogas facilitan el riesgo o perjudican la protección de consumo. Se concluye que el profesional de enfermería debe aplicar los conceptos de autoeficacia y actitud hacia el consumo para orientar las acciones de promoción de la salud y prevención del consumo de drogas en niños y adolescentes.

Descriptores: Eficacia; Actitud; Prevención Primaria; Trastornos Relacionados con Sustancias, Enfermería.
\end{abstract}

\footnotetext{
${ }^{1}$ Maestría en Enfermería, Profesor, Facultad de Enfermería, Universidad de Ciencias Aplicadas y Ambientales, U.D.C.A, Colombia. E-mail: dormelo@udca.edu.co.

${ }^{2}$ Enfermeira, Doctor em Enfermagem, Profesor Doctor, Escola de Enfermagem de Ribeirão Preto, Universidade de São Paulo, Centro Colaborador de la OMS para el Desarrollo de la Investigación en Enfermería, SP, Brasil. E-mail: lucila@eerp.usp.br.
}

Correspondencia:

Lucila Castanheira Nascimento

Universidade de São Paulo. Escola de Enfermagem de Ribeirão Preto

Av. Bandeirantes, 3900

Bairro Monte Alegre

CEP: 14040-902 Ribeirão Preto, SP, Brasil

E-mail: lucila@eerp.usp.br 


\title{
Autoeficácia e atitude frente ao consumo de drogas na infância: explorando conceitos
}

O objetivo deste trabalho foi explorar os conceitos de autoeficácia e de atitude frente ao consumo de drogas, derivados da teoria da motivação e do comportamento humano. Tratase de reflexão teórica, construída com base na literatura. Altos níveis de autoeficácia têm consequências benéficas para o funcionamento do indivíduo. Em relação à atitude para o consumo das drogas, a presença ou ausência de preconceitos e valores positivos frente às drogas facilitam o risco ou proteção para o consumo. Conclui-se que o enfermeiro deve aplicar os conceitos de autoeficácia e a atitude para o consumo para orientar as ações de promoção de saúde e prevenção do consumo de drogas nos escolares.

Descritores: Eficácia; Atitude; Prevenção Primária; Transtornos Relacionados ao Uso de Substâncias; Enfermagem.

\section{Self-efficacy and Attitudes Towards Drug Consumption in Childhood: Exploring Concepts}

\begin{abstract}
This paper aims to explore the concepts of self-efficacy and attitude towards consumption, approached by the theory of motivation and human behavior. This is a theoretical study based on literature. High levels of self-efficacy have beneficial consequences for the functioning of the individual. Concerning attitudes towards drugs, the presence or absence of preconceptions and positive values of drugs increases risk or consumer protection. Nurses should apply the concepts of self-efficacy and attitudes towards consumption to guide actions of health promotion and prevention of drug consumption in children and adolescents.
\end{abstract}

Descriptors: Efficacy; Attitude; Primary Prevention; Substance-Related Disorders; Nursing.

\section{Introducción}

El alcance del consumo de drogas es un indicador importante de la magnitud del problema mundial de las drogas. La cifra total de consumidores de drogas en el mundo se calcula en 200 millones de personas ${ }^{(1)}$. En Colombia, el consumo de sustancias psicoactivas por los jóvenes escolares ha aumentado, con edades de inicio cada vez más tempranas. El Observatorio de Drogas de Colombia (ODC) muestra que hay cada vez más niños y niñas que experimentan Sustancias Psicoactivas (SPA); mientras el estudio de 1996 mostraba un promedio de inicio con el alcohol de 15,9 años, el estudio de Rumbos en el año 2001 mostró un inicio a los 12,9 años, aunque el $15,2 \%$ inició antes de los 10 años ${ }^{(2)}$. Las prevalencias más altas corresponden al consumo de drogas lícitas, tales como el alcohol y cigarrillo, presente en todos los grupos de edad estudiados y en proporción creciente a medida que aumenta la edad; los valores van desde
$50 \%$ a $20 \%$ en escolares de 12 y 13 años, hasta $92 \%$ y $67 \%$ en el grupo de 18 a 19 años, para cada sustancia, respectivamente. No se evidencian diferencias en el consumo de alcohol y cigarrillo entre los colegios públicos y privados $^{(1)}$. Dentro de las drogas ilícitas, la marihuana es la sustancia que presenta las mayores prevalencias a nivel nacional; su consumo es significativamente mayor entre hombres que en mujeres y entre estudiantes que han tenido problemas de comportamiento y/o disciplina en el colegio y son repitentes y pertenecientes a colegios públicos $^{(1,3)}$.

De acuerdo con el último estudio de Vigilancia Epidemiológica de Sustancias Psicoactivas (VESPA) de la Secretaría Distrital de Salud (SDS) de Bogotá, entre enero y septiembre del año 2006, los centros de atención para adicción en Bogotá reportaron 3.461 casos de niños y jóvenes en tratamiento por alcoholismo 
y drogadicción. La gran mayoría de menores se inician con el consumo de bebidas alcohólicas entre los 10 y 13 años $y$, aproximadamente a los 12 años, consumen cigarrillo y marihuana. El mayor consumo se encuentra entre estudiantes y desempleados y se concentra entre las edades de 11 a 25 años. El 56,9\% de consumidores se encuentra en la educación básica secundaria(4).

Los factores de riesgo asociados a la percepción de riesgo, las actitudes y creencias facilitan el inicio en el consumo de drogas. Estos factores son la actitud favorable hacia el consumo de drogas, las creencias positivas acerca de las consecuencias, las influencias normativas, la intención de consumo y la percepción de riesgo(5). Los jóvenes de hoy tienen percepciones propias, perciben el mundo en formas diferentes $y$, por lo tanto, es necesario conocer el sentido que le dan al uso de drogas y a los significados que dan a su prevención(6).

No existe consenso para señalar a una teoría o modelo como el más idóneo en la explicación y aproximación del consumo de drogas en la infancia. Dentro de las teorías que han intentado comprender el consumo de drogas, se encuentran la teoría del Aprendizaje Social y la Teoría de la Acción Planeada(7). Derivada de la Teoría de Aprendizaje Social, se encuentra la autoeficacia, que consiste en creer en las propias capacidades para organizar y ejecutar las acciones necesarias para manejar situaciones futuras. La Teoría de la Acción Planeada (TAP) plantea que hay varios factores que anteceden y explican el comportamiento humano. Concretamente, trata de explicar las conductas que están bajo el control consciente de los individuos, a partir de distintos determinantes que la preceden y la explican ${ }^{(7)}$. La prevención del consumo de drogas debe fundamentarse sobre el conocimiento científico en torno al origen y desarrollo de este comportamiento. Sin embargo, en la práctica, muchas veces, la prevención se realiza más en torno a la urgencia de implementar medidas que basada en un análisis concienzudo de la aplicación teórica ${ }^{(8)}$.

De acuerdo con el informe de gestión 1999-2001 de la Secretaría de Salud de Bogota, las acciones de prevención estaban, en su mayoría, concentradas en ofrecer información acerca del consumo y sus consecuencias. Dicho informe denota la insistencia marcada en un factor de riesgo y su diseño pretende la cobertura masiva, ignorando las particularidades de ciertos segmentos de la población adolescente ${ }^{(9)}$.

En los últimos 20 años se ha venido insistiendo sobre la prevención como estrategia eficaz para hacer frente al consumo de drogas. En Colombia ha faltado una mayor claridad y consenso sobre los modelos teóricos que orientan las acciones preventivas ${ }^{(10)}$. La poca evaluación realizada en estos programas han mostrado que las acciones de quienes conciben e implementan los programas de prevención lo hacen desde imaginarios sociales, tomados más de la tradición que de una fundamentación científica y técnica(10). En ese sentido, desde la visión de las autoras, el consumo de drogas en adolescentes y jóvenes debe ser abordado desde un punto de vista comprensivo que permita conocer los motivos por los cuales los sujetos consumen drogas. El objetivo de este artículo de actualización es explorar los conceptos de autoeficacia y actitud hacia el consumo desde las teorías de la motivación y comportamiento humano, que permitan comprender los significados del uso de drogas y sean aplicados por el profesional de enfermería para orientar el diseño de programas de prevención del consumo de drogas, ajustados a la situación real de los adolescentes y jóvenes y, con ello, contribuir a la reducción de la demanda de drogas.

\section{Explorando los conceptos: La conducta humana}

El comportamiento humano es directamente observable, lo que no ocurre con los procesos psicológicos que se desencadenan antes, mientras o después de la ejecución del mismo. Se trata de comprender, explicar el comportamiento, y predecirlo con anterioridad a que éste se realice(11). Cuando se alude a la motivación por lo general se hace referencia al por qué, o al cómo se hace o se deja de hacer algo, teniendo en cuenta las condiciones internas y externas. La motivación permite la descripción y explicación del comportamiento humano, teniendo en cuenta no sólo los factores que causan y determinan el proceso de la conducta(12). El análisis de los procesos motivacionales está posibilitando la comprensión de las causas de actividades y de actos inadecuados, que van contra la salud o contra la ley, como la adicción y la delincuencia(13). La importancia de la motivación se relaciona en cómo se la mire, ya sea como variable independiente causal del comportamiento o como variable dependiente de otros factores que permiten predecirla y controlarla(14).

La conducta de consumo se refiere a la que se adquiere y mantiene seguir un modelo, refuerzo social, efectos anticipatorios de la droga, experiencia directa de sus efectos como refuerzo, castigo físico y dependencia física. Algunos determinantes importantes son los eventos vitales estresantes, presiones, trabajo, 
familia, papel de las redes sociales y el apoyo social que tiene el sujeto. Esto varía a lo largo del tiempo, como la influencia de un individuo a otro. La decisión última de consumir o no, esta en función de las expectativas de autoeficacia y del resultado que tiene la persona en el contexto situacional en que se encuentra. Los efectos anticipados de una conducta son los que parecen importantes a la hora de determinar si esta conducta se realiza o no. Algunos autores han observado el papel concreto de las expectativas de resultado positivo en relación con el consumo de drogas y sus efectos sobre la conducta(15).

\section{Autoeficacia}

La teoría de la autoeficacia es una propuesta hecha por Albert Bandura como modelo unificador para explicar el cambio conductual. Interpreta la motivación como una actividad que se manifiesta de forma espontánea, sin necesidad de reducirla a una mera reacción ante un estimulo especifico. Considera a la conducta motivada como propositiva porque se desarrolla impulsada por planes, metas y objetivos. Las creencias sobre nuestra autoeficacia influyen sobre nuestro modo de pensar, sentir, motivarnos y actuar. De manera que si alguien ha desarrollado la convicción en su propia capacidad de superar los retos que le plantea la vida, pensará que los puede superar, se sentirá seguro a la hora de emprender una tarea, la motivación para el esfuerzo que tendrá que desarrollar será alta y llevará a cabo las acciones necesarias para conseguirlo(16). Esta teoría contempla dos conceptos: La expectativa de autoeficacia y la expectativa de resultado. La autoeficacia alude a la creencia de la persona acerca de poseer o no las condiciones (habilidades) requeridas para ejecutar una acción. El resultado alude a la creencia de la persona de que esa acción si conduce a ese resultado; el reforzamiento alude al incentivo que el resultado esperado representa para la persona(14). Tanto las expectativas de autoeficacia como de resultado, intervienen en la habilidad de una persona para afrontar situaciones de alto riesgo. Dichas expectativas están influenciadas por creencias sociales y culturales, por la autoestima del individuo, por factores ambientales y por el condicionamiento del deseo. Las expectativas de la consecuencia positiva son los determinantes del uso del alcohol y otras sustancias ${ }^{(15)}$.

Las expectativas de eficacia influyen tanto en la iniciación, como en la persistencia y regulación de la conducta, que surgen o se adquieren de la experiencia directa e indirecta, de la persuasión verbal y del estado fisiológico(12,14). La expectativa de autoeficacia es el principal proceso que determina el inicio de un proceso de enfrentamiento, la cantidad de esfuerzo que la persona invierte en la ejecución de la conducta, y el mantenimiento en la acción a pesar de los obstáculos. Esa operación de la expectativa de autoeficacia tiene lugar siempre y cuando la persona posea habilidades requeridas para la acción y ésta conduzca a los incentivos adecuados ${ }^{(14,17)}$. La autoeficacia percibida se refiere a la capacidad que la gente cree tener para ejercer control sobre su motivación y comportamiento y sobre su ambiente social(12). La autoeficacia constituye el proceso que, no solamente, guía la selección de metas (intencionalidad) por parte del individuo, sino que también determina la cantidad de esfuerzo o voluntad que el individuo va a invertir después en el esfuerzo realizado para lograr la meta y la persistencia en la misma(14).

Hay una relación entre autoeficacia y las conductas de salud específicas. Se ha utilizado la percepción de autoeficacia en distintas áreas como las de elección vocacional, comportamiento saludable, funcionamiento físico, consecución de metas, manejo de enfermedades crónicas, uso de drogas, actividad sexual, fumar, realizar ejercicio, bajar de peso, y también la habilidad para recuperarse de los problemas de salud o evitar potenciales riesgos para la salud. Altos niveles de autoeficacia tienen consecuencias beneficiosas para el funcionamiento del individuo y su bienestar(18-19).

Autores mencionan que la autoeficacia es una variable de predicción para el consumo de drogas y ha demostrado ser un factor protector para el consumo de drogas(17). Así, los jóvenes que no consumen tabaco ni alcohol reportan altos niveles de autoeficacia $y$ autoestima; los sujetos que puntúan alto en autoeficacia mantienen niveles bajos de consumo de alcohol y estos niveles de autoeficacia modulan las habilidades y el consumo de drogas. La autoeficacia es el factor de mayor peso para lograr el abandono o disminución del hábito de fumar y las puntuaciones altas en autoeficacia están asociadas a la convicción por parte de los sujetos de sentirse capaces de mantener la abstinencia en el momento de iniciar el tratamiento(15,20-21).

Niveles bajos de autoeficacia son factor de riesgo para el consumo de drogas. Los drogadictos tienen puntuaciones bajas de autoeficacia y por tanto están mas expuestos a no resistir la tentación del consumo, esto sugiere que con menor autoeficacia la intención es más fuerte para el mantenimiento del consumo(15). Si los jóvenes creen que beber o consumir otro tipo de 
sustancias les proporciona ventajas importantes para ellos, la autoeficacia se dirige a las conductas necesarias para experimentar o iniciar el habito. Los adolescentes con baja eficacia percibida se implican más en actividades de consumo de drogas, conductas sexuales de riesgo y conductas delictivas. El motor principal de la conducta delictiva es la percepción de autoeficacia(18,22-23). Pero en el caso de que quieran abandonar el hábito, si perciben que el consumo les proporciona ventajas importantes, se pone en peligro la eficacia relativa a la propia habilidad para abandonarlo. Los conflictos de este tipo, como iniciarse en el consumo o no, o entre abandonar o no abandonar, caracterizan de forma única el problema de la conducta adictiva(15). Es así como las actividades de prevención deben ser ejecutadas con mucha precaución, debido a que en lugar de fomentar la autoeficacia se puede estar estimulando el consumo de drogas.

La edad de inicio del consumo predice el posterior consumo de sustancias. Aquellos que iniciaron su consumo de alcohol a edad más temprana, tienen mayor probabilidad de presentar en la actualidad un consumo regular de bebidas alcohólicas y mayor frecuencia que aquellos que se iniciaron más tarde ${ }^{(24)}$. La adolescencia se acompaña de un progresivo descenso de la autoeficacia, los estudios mostraron que los niños más pequeños muestran mayor nivel de autoeficacia mientras que los grupos de mayor edad muestran niveles menores ${ }^{(18)}$.

La autoeficacia se convierte en un constructo importante que debe ser tenido en cuenta para guiar las acciones de promoción de la salud y prevención del consumo de drogas en escolares. Si se tiene en cuenta este concepto se podrá conocer previamente la capacidad que tienen los escolares para iniciarse en el consumo de drogas; es necesario incentivar la autoeficacia desde la más temprana edad y potencializarla en los grupos de adolescente con el fin de aumentar la capacidad del sujeto para afrontar los riesgos que la vida le presenta relacionados con el consumo de drogas.

\section{Actitud}

Se considera que muchos de los comportamientos humanos de la vida diaria se encuentran bajo control volitivo. Los factores psicológicos contemplan características afectivas (estados de ánimo y emociones) y cognitivas (creencias y expectativas) y los factores sociales contemplan las redes y normas sociales. Estos dos factores actuarán facilitando o inhibiendo la manifestación de una dada conducta y deben ser considerados a la hora de predecir la aparición, el mantenimiento o la extinción de una conducta en un contexto determinado. La mayoría de las investigaciones que se han centrado en el estudio de los distintos factores que pueden predecir el comportamiento han dado relevancia especial a los factores cognitivos, y, especialmente, a las actitudes ${ }^{(11)}$.

La Teoría de la Acción Razonada (TAR) y replanteada como Teoría de Acción Planeada (TAP) trata de explicar las conductas que están bajo control consciente de los individuos, a partir de distintos determinantes que la preceden y la explican. El determinante inmediato de la conducta no es la actitud propiamente dicha, sino la intención de realizarla ${ }^{(11,14)}$. La actitud viene determinada por cada una de las creencias que la persona tiene hacia el objeto (sea cosa, persona o institución) y la evaluación positiva/negativa realizada hacia cada una de esas creencias. Esta evaluación es el componente afectivo de la actitud, determinando la motivación y la fuerza de la intención de conducta. Se pueden poseer distintas creencias pero éstas, por sí solas, no conducen a la acción. Una evaluación alta de las mismas por parte de un individuo indica la importancia que tienen para él y el grado de compromiso con ellas ${ }^{(12,14)}$

Los distintos elementos que influyen en el inicio de una acción conducen a valorar las distintas creencias que están en juego, y no a una creencia general, ya que no se otorga el mismo grado de aceptación o rechazo a cada uno de los componentes específicos que la conforman. El conocimiento del mayor número de creencias específicas sobre la conducta, conjuntamente con el efecto de la valoración de las mismas, va a permitir una mejor predicción de la actitud y, por lo tanto, de la intención concreta de llevarla a término(11). El conocimiento sobre las creencias específicas de lo que los otros piensan de cada uno de los comportamientos específicos (hábito de fumar, práctica de ejercicio físico, alimentación sana, etc. ) va a influir en la intención de llevar a cabo o no una conducta general (mantenimiento de salud), siempre en función de la motivación para complacerles(11)

Laactitud seencuentra en función delasconsecuencias personales, es decir el costo beneficio y el valor afectivo que se le de a esas consecuencias. La baja percepción de riesgo genera un mayor peligro para el uso de drogas, ya que por un lado los adolescentes creen que las drogas no son tan peligrosas y se creen capaces de dejarlas en cualquier momento(25). Por ejemplo los adolescentes le dan mayor valor a las sensaciones placenteras y a los cambios en el estado de ánimo(26). Aquellos que consumen mayor cantidad de alcohol perciben más resultados positivos en la práctica de actividades riesgosas que aquellos que consumen menos ${ }^{(24)}$. 
Frente a la actitud hacia las drogas se encontró que la presencia o ausencia de prejuicios y valoraciones positivas sobre las drogas facilita el riesgo o protección de consumo(26). El alcohol y el tabaco son las drogas de las que se ha escuchado más por los adolescentes pero, a su vez, las estiman menos peligrosas ${ }^{(27)}$. Si se percibe una droga como riesgo y se valora su consumo como peligroso, la probabilidad de convertirse en consumidor o de pasar a consumos compulsivos o intensos será mucho menor. Los adolescentes que tienen más expectativas positivas frente al alcohol, consumen mayores cantidades de alcohol que sus compañeros que tienen menos expectativas positivas hacia el alcohol(24). Por otro lado en los espacios públicos no hay un seguimiento de la normativa legal ni ningún tipo de censura, por tanto se convierten en ambientes facilitadores para el consumo. El tiempo libre y la intención de fumar en mujeres y hombres se asocia con actividades compartidas con el grupo de amigos, entre los hombres son bares, discoteca y fiestas, mientras que para las mujeres la cafetería, el cine, y la calle. Aquellos que solían ir al cine presentaban menor consumo de tabaco y también de alcohol que los que acostumbraban salir a bares y discotecas ${ }^{(28)}$.

Con respecto al control de consumo, se encontró que los adolescentes clasifican algunas sustancias como menos peligrosas y ellos mismos se atribuyen una mayor capacidad de control sobre el consumo cuando se hace de manera recreativa u ocasional. Los que logran una mayor autorregulación tienen una menor vulnerabilidad, evitan exponerse a situaciones de riesgo o pueden presentar un consumo exploratorio y esporádico y no avanzar hacia la dependencia(26,29). Con respecto a la edad, se encontró que entre aquellos que inician su consumo a más temprana edad tienen más probabilidades de ser consumidores más tarde y de presentar un elevado consumo de alcohol(24). Las actitudes permisivas se incrementa con la edad y las negativas del alcohol y de valorar la acción preventiva disminuyen con la edad; conforme aumenta el grado escolar y la edad, es mayor la oferta y el consumo, el cual se encuentra asociado con la permisividad social(30).

La acción familiar y la educación secundaria parecen fracasar en el fortalecimiento de las actitudes positivas que inhiben el consumo de alcohol durante la adolescencia, la permisividad y la aceptación social son factores macrosociales vinculados al incremento del consumo(27,30). La imitación del comportamiento en relación con patrones de consumo junto a la permisividad facilita que los niños registren niveles de ingesta; al $60 \%$ de los niños que consumieron drogas, se les fue ofrecido por primera vez la sustancia en un contexto hogareño(27).

\section{Conclusiones}

La autoeficacia y la actitud se convierten en constructos importantes a tener en cuenta por los profesionales de enfermería con el fin de dirigir los programas de promoción y prevención y que estos impacten positivamente en la población escolar. Los individuos que se perciben con baja autoeficacia tienen un repertorio escaso de conductas que los proteja contra el consumo de drogas, beben en exceso y tienen creencias de que el consumo los ayuda a experimentar relajación. Individuos con bajas puntuaciones en autoeficacia asociado a la valoración de ventajas positivas del consumo, consumen más alcohol y más frecuentemente que aquellos con puntuaciones altas de autoeficacia.

El consumo de drogas es una problemática producto de la globalización, que abarca dimensiones muy amplias y complejas. Es una situación que la enfermera debe enfrentar desde la promoción de la salud y la prevención, llevando a cabo proyectos y programas que ayuden a disminuir la demanda de drogas y a mejorar la calidad de vida, partiendo de la investigación y abordando el consumo de drogas en los niños escolares con el fin de obtener información real, que permita identificar su situación de salud y plantear nuevas estrategias de promoción de la salud y prevención integral, que impacten en la calidad de vida de los niños, sus familias, escuela y comunidad.

Los programas de prevención del consumo de drogas deben incluir el fomento de la autoeficacia, favoreciendo el desarrollo de habilidades sociales, el enfrentamiento de las diferentes situaciones de la vida y la toma de decisiones. Pero también es necesario que dichos programas tengan un modelo teórico que permita plantear objetivos y metas concretas que sean propias de los ambientes psicosociales y culturales donde estos se desarrollan, con el fin de lograr los resultados esperados y no de incentivar conductas de riesgo como el uso de drogas. Estos programas deben contemplar a todos los miembros involucrados y no sólo a los escolares, dirigiendo las acciones hacia los profesores, la familia y comunidad en general, contemplando dentro de ellas a todas las instituciones públicas o privadas que tienen relación con los escolares.

Teniendo en cuenta que la edad de inicio del consumo de drogas tanto licitas como ilícitas ha ido disminuyendo paulatinamente en nuestro país, los 
escolares se convierten en foco de atención de los programas dirigidos a disminuir el consumo de drogas, ya que lo que se hace en esta etapa de la vida repercute más adelante en las acciones de la adolescencia. Los niños en edad escolar atraviesan por una etapa en la que lo importante es el desarrollo de destrezas sociales, es una etapa de calma emocional pero sus intereses van dirigidos al medio externo o mundo social.

En el marco de los 30 años de la Atención Primaria de Salud (APS) es necesario que la enfermera retome sus experiencias frente a las acciones de promoción de la salud y reoriente sus responsabilidades en la atención primaria. Estos programas deben contemplar aspectos diagnósticos, identificando la situación real de las comunidades y planteando estrategias de intervención que involucren al escolar, su familia, la escuela, la comunidad y los sectores económicos, educativos y de salud, permitiendo la integración de los sistemas sociales, intersectoriales y la comunidad, incentivando la participación comunitaria y la educación en salud, que repercuta en la calidad de vida de los escolares.

\section{Agradecimientos}

Agradecemos a la Comisión Interamericana para el Control del Abuso de Drogas/CICAD de la Secretaria de Seguridad Multidimensional/SSM de la Organización de los Estados Americanos/OEA, la Secretaria Nacional de Políticas sobre Drogas/SENAD do Gabinete de Seguridad Institucional/Brasil, la Escuela de Enfermería de Ribeirao Preto de la Universidad de Sao Paulo y Centro Colaborador de la Organización Mundial de la Salud para el Desarrollo de la Investigación en Enfermería, la población representada en los estudios de investigación, bien como a las autoridades de las universidades representadas por los participantes del Programa En-Line de Especialización en Investigación sobre el Fenómeno de las Drogas PREINVEST, periodos 2005, 2006, 2007 y 2008.

\section{Referencias}

1. Pérez A. Transiciones en el consumo de drogas en Colombia. Ministerio del Interior y de Justicia. Dirección Nacional de Estupefaciente. Bogotá: Corporación Nuevos Rumbos; 2007. [Acceso 24 de Mayo de 2008] Disponible en: http:// w3.nuevosrumbos.org/documentos/Transiciones_libro_ Agosto2007.pdf

2. Dirección Nacional de Estupefacientes, Observatorio de Drogas de Colombia: Acciones y Resultados 2003; 2004. [acceso en: 13 Junio 2007] Disponible en: http://www.dne.gov. $\mathrm{co} /$ ? idcategoria $=834$

3. Alcaldía de Medellín (CO). Secretaría de Salud. ESE Carisma. Consumo de Drogas en Jóvenes Escolarizados del Municipio de Medellín. 2007. [acceso en: 26 Julio de 2008]. Disponible en: http://www.esecarisma.gov.co/paginas/Comite_prevendrogas/ investigacion_completa_medellin.pdf

4. Alcaldía de Bogotá (CO). Portal Bogotá. Menores consumen sustancias psicoactivas desde los diez años. 9 de mayo de 2007. [acceso en: 27 Noviembre 2008]. Disponible en: http:// www.bogota.gov.co/portel/libreriha/pp/frame_detalle.php?h_ id $=18601$ \&patron $=01.11$

5. Martínez E. IATROGENIA De las buenas intenciones... Al daño preventivo. En: Prevención basada en la evidencia: Comunidades y Drogas. [Internet] Marzo 15 y 16 de 2007; Bogotá. AVISPA. [Acceso 26 de Abril del 2008] Disponible en: http://w3.nuevosrumbos.org/documentos/Ponencia_Efren_ Martinez.pdf

6. Ghiardo F. Acercándonos al Sentido del Uso de Drogas y la Prevención Desde los Jóvenes. Rev Ultima Década [Internet] abril 2003. [Acceso 10 de Mayo 2008] Disponible en: http://148.215.1.166:89/redalyc/pdf/195/19501806.pdf

7. Centro de Integración Juvenil A. C. Teorías y Modelos Explicativos del Consumo de Drogas. 2007. México. [acceso en; 25 Noviembre de 2008] Disponible en: http://www.cij. gob.mx/Paginas/MenuIzquierdo/InformacionPara/Especialistas/ Prevencion/InvPreventiva/teoriasmodelosasp
8. Perea I. Prevención especifica del Consumo de Drogas. Artículos de Prevención. Juventud y Prevención Integral en Línea 2006 [acceso en: 25 Noviembre 2008] Disponible en: http:// www.juventudyprevencion.com/main.html

9. Londoño C. Modelo Cognitivo-Social Integrado para la Prevencion del Abuso en el Consumo de Alcohol. Típica Bol Electrónico Salud Escolar [Internet] 2007 [acceso en: 15 abri 2009]; 3(2). Disponible en: http://www.tipica.org/index. php?option $=$ com_content\&view $=$ article\&id $=73 \&$ Itemid $=83$

10. Calderon GA, Castaño GA, Parra AM. Estado del Arte sobre Investigaciones en Prevencion de la Farmacodependencia y Prevalencia del Consumo en Colombia (1980-2002). Organizador Fundación Universitaria Luis Amigo (FUMLAN). XII Congreso Colombiano de Psicología, Paz y sociedad; 3-6 de mayo 2006; Medellín-Antioquia, Colombia [acceso en: 15 abril 2009] Disponible en: http://grupodeinvestigacionenadicciones. blogspot.com/2008/10/proyectos-del-grupo.html

11. Carpi A, Breva A. La Predicción de la Conducta a través de los Constructos que Integran la Teoría de Acción Planeada. Rev Electrónica Motivación y Emoción R.E.M.E. [Internet] Mayo 2001 [acceso en: 27 julio 2008]; 4(7). Disponible en: http://reme.uji. es/articulos/abreva7191302101/texto.html

12. Córdoba A. La Cognición y la Motivación Humana. Boletín Electrónico de salud Escolar. Típica. [Internet] Julio-Diciembre 2006 [acceso en: 26 Abril 2008] 2(2): 1-22 Disponible en http://www.tipica.org/pdf/03_cordoba_cognicion_motivacion_ humana.pdf

13. Utria O. La Importancia del Concepto de Motivación en la Psicología. Rev Digital Psicol. Suma Digital Psicol 2007; 2(3):55-78.

14. Flórez L. El desarrollo de la conducta saludable: un proceso segmentado por etapas. En

Psicología Social de la Salud: Promoción y Prevención. Bogotá: Manual Moderno; 2007. p. 90-82. 
15. López-Torrecillas F, Salvador MM, Verdejo A, Cobo P. Autoeficacia y Drogas: Una Revisión. Sicopatol Clín, Legal Forense 2002; 2(1):33-51.

16. Barberà E. Modelos Explicativos en Psicología de la Motivación. Rev Electrónica Motivación y Emoción. R.E.M.E. [Internet] Mayo 2002. [acceso en: 27 Julio 2008] 5 (10). Disponible en: http:// reme.uji.es/articulos/abarbe7630705102/texto.html

17. Martínez R, Pedrão LJ, Alonso MM, López KS, Oliva NN. Autoestima, Autoeficacia Percibida, Consumo de Tabaco y Alcohol en Estudiantes de Educación Secundaria de Area Urbana y Rural de Monterrey, Nuevo León, México. Rev. LatinoAm. Enfermagem [internet]. 2008 [acceso en: 7 Mayo 2010] 16(especial) Disponible en: http://www.scielo.br/pdf/rlae/ v16nspe/es_18.pdf

18. Carrasco MA, Del Barrio MV. Evaluación de la Autoeficacia en Niños y Adolescentes. Psicothema 2002; 14 (2):323-32.

19. Olivari C, Urra E. Autoeficacia y Conductas de salud. Cienc Enferm 2007; 13(1):9-15.

20. Pérez A, Clemente L, Hernández MA, Morales C. La Autoeficacia como método de deshabituación tabáquica. Anales de Psiquiatría. [Internet] Enero 2001. [acceso en: 23 Noviembre 2008]; 17(1). Disponible en http://www.grupoaran.com/WEB/ ediciones $/$ revistas $/ 003$. asp? $\mathrm{IR}=26 \&$ fechaPublicacion $=01 / 01 / 20$ $01 \& I D A R=569 \& I D T A=6 \& I E S P=16 \& V O L=17 \& N U M=1 \& i D N R=63$

21. López-Torrecillas F, Peralta I, Muñoz M, Godoy J. Autocontrol y consumo de drogas. Adicciones. [Internet] 2003 [acceso en: 26 Julio 2008]. 15(2): 127-36. Disponible en: http://www. adicciones.es/files/03-Autocontrol\%20y\%20consumo.pdf

22. Garrido E, Herrero C. Masip, J. Autoeficacia y Delincuencia. Psicothema. [Internet] 2002 [acceso en: 26 Julio 2008]. 14 (suplem.1): 63-71. Disponible en: http://www.psicothema.es/ pdf/3475.pdf

23. Lopez Karla López KS, Lobo da Costa M. Conducta Antisocial y Consumo de Alcohol en Adolescentes Escolares. Rev. LatinoAm. Enfermagem [Internet]. 2008. [acceso 7 Mayo 2010] 16(2). Disponible en: http://www.scielo.br/pdf/rlae/v16n2/es_20.pdf
24. Cassola I, Pilatti A, Alderete A, Godoy JC. Conductas de riesgo, expectativas hacia el alcohol y consumo de alcohol en adolescentes de la ciudad de Córdoba. Evaluar 2005; 5:38-54.

25. Bolaños H, Falleiros D, Carvalho MDG, Lossi MA. Opiniones de los Adolescentes Escolares sobre Consumo de Drogas: Un Estudio de Caso en Lima, Perú. Rev. Latino-Am. Enfermagem [internet]. 2008. [acceso en: 26 Noviembre 2008] 16 (especial). Disponible en: http://www.scielo.br/pdf/rlae/v16nspe/es_08.pdf

26. Cáceres D, Salazar I, Varela M, Tovar J. Consumo de Drogas en Jóvenes Universitarios y su Relación de Riesgo y Protección con los Factores Psicosociales. Univ Psicol. [Internet]. Octubre-Diciembre 2006 [acceso en: 26 Julio 2008] 5(3): 521-34. Disponible en: http://sparta.javeriana.edu.co/ psicologia/publicaciones/actualizarrevista/archivos/V5N306_ consumodrogas.pdf

27. Obando P, Saenz MA. Percepción, consumo y factores asociados con el fenómeno droga en población escolar de Heredia, Costa Rica. Adicciones. 2000; 12(1):127-35.

28. Nebot M, Tomas Z, Ariza C, Valmayor S, Muddea A. Factores asociados con la intención de fumar y el inicio del hábito tabáquico en escolares: resultados del estudio ESFA en Barcelona. Gac Sanit. 2002;16(2):131-8.

29. Rodríguez E, DiazD, Gracia SE, Guerrero JA, Gómez EL. Capacidad Predictiva de la Teoría de la Conducta planificada en la intención y uso de drogas ilícitas entre estudiantes mexicanos. Salud Mental. [Internet]. 2007 [acceso en: 25 Noviembre 2008] 30 (1). Disponible en: http://www.imbiomed.com.mx/1/1/ articulos.php? method $=$ showDetail\&id_articulo $=43516 \&$ id seccion=1169\&id_ejemplar=4422\&id_revista $=1$

30. Moreno J. Valores, actitudes hacia el alcohol y consumo en adolescentes varones. Limite. Rev Fil Psicol. 2006;1(13):195-221.

Recibido: 17.8 .2009

Aceptado: 12.5 .2010 